comparative study of magnetic resonance imaging and electroencephalography in 34 children with tuberous sclerosis. Epilepsia Nov/Dec 1990;

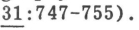

OOMMENT. The MRI is more efficient than CT in detecting and localizing tubers of tuberous sclerosis which appear as high intensity signal areas in T2-weighted sequence. The cortical tubers shown on MRI are epileptogenic whereas subependymal calcifications as detected by CT are non-epileptogenic. Frontal lesions present from the first year of life often have a delayed EEG expression, in keeping with the posteroanterior migration of epileptic foci in childhood, the result of maturational changes.

\title{
VON HIPPEL-LINDAU DISEASE AND GADOLINIUM MRI
}

Gadolinium-enhanced MRI was used to determine the frequency and distribution of CNS lesions in 50 patients with Von Hippel-Lindau Disease (VHL) at the National Institutes of Health, Bethesda, MD; University of Louisville, KY; and the Massachusetts General Hospital, Boston, MA. CNS hemangioblastomas were found in 36 (72\%). The cerebellum was affected in $52 \%$, spinal cord in $44 \%$, and the brainstem in $18 \%$. One-half of these tumors were asymptomatic and without clinical signs. The youngest patient was 11 years old and asymptomatic. The youngest symptomatic patient with $\mathrm{VHL}$ was 2 years of age with symptoms of ocular lesions. The mean age of all VHL patients was 34 years with a range of 11-62 years. (Filling-Katz $\mathbb{R}$ et al. Central nervous system involvement in Von Hippel-Lindau disease. Neurology Jan 1991; 41: 41-46).

COMMENT. Hemangioblastomas and VHL may be detected by 11 years of age and the screening of patients at-risk for VHL should begin at 11 years using gadolinium-enhanced MRI; ophthalmic examination should be initiated within the first two years of life. Diagnosis of VHL is important for genetic counseling as well as early detection of unsuspected ocular and visceral tumors which may lead to blindness or early death unless treated.

SUBARACHNOID METASTATIC TUMORS AND MRI

Subarachnoid metastatic disease was evaluated in 17 children with primary intracranial neoplasms at the Departments of Neurology and Radiology, The Children's Hospital of Philadelphia, PA. The tumors were principally neuroectodermal, ependymomas, and astrocytomas and the majority originated in the posterior fossa. The gadolinium MRI was superior in delineating spinal cord nodules and "sugar coating" whereas myelography and CT more readily revealed nerve root sleeve filling defects. (Kramer ED et al. Comparison of myelography with CT followup versus gadolinium MRI for subarachnoid metastatic disease in chil-

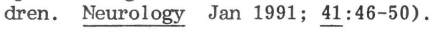

COMENT. Gadolinium-enhanced MRI appears to be a safe non-invasive test and the technique of choice for the diagnosis of subarachnoid metastatic disease. 